\title{
EviPrev, ein Programm zu Prävention und Gesundheitsförderung in der Hausarztpraxis
}

Jacques Cornuz ${ }^{a}$,

Nicolas Rodondi ${ }^{a}$,

Ruth Ospelt ${ }^{b}$, Marco Zoller ${ }^{c}$,

Dominique Durrer,

Erich Tschirky ${ }^{b}$

Andreas Biedermann ${ }^{b}$

a PMU Policlinique Médicale Universitaire, Lausanne

b PHS Public Health Services $\mathrm{GmbH}$, Herzogenbuchsee

c Institut für Hausarztmedizin, Universität Zürich

d Arbeitsgruppe Prävention des Kollegiums für Hausarztmedizin (KHM), Bern
Korrespondenz: Verein EviPrev c/o Andreas Biedermann Güterstrasse 12 CH-3360 Herzogenbuchsee Tel. 0629611001

\section{biedermann@}

public-health-services.ch
Kürzlich tat sich eine Gruppe aus Ärztinnen und Ärzten und Präventionsspezialisten zusammen, um in Zusammenarbeit mit Hausärzten das Projekt EviPrev zu lancieren; die Abkürzung steht für Evidence based preventive medicine. Das Programm will schweizerischen Ärztinnen und Ärzten (Hausärzte und Fachärzte), die dies wünschen, neue Tools für ihre Interventionen im Bereich der Prävention zur Verfügung stellen. Dieser Artikel fasst die wichtigsten Ziele und die Rahmenbedingungen für die Entwicklung des Programms zusammen.

\section{Ausgangslage}

In der Schweiz sind drei Viertel der Todesfälle und mehr als die Hälfte der verlorenen Lebensjahre auf chronische, nicht-übertragbare Krankheiten zurückzuführen (Herz-Kreislauf-Krankheiten, Diabetes, Krebs und chronische Lungenleiden) [1-4] (Abb 1). Die hauptsächlichen Risikofaktoren sind Tabakkonsum, eine unausgewogene Ernährung, Bewegungsmangel und Alkoholmissbrauch. Diese Faktoren spielen auch eine wichtige Rolle bei der Sekundärprävention. So hat bei koronaren Herzkrankheiten die Tabakentwöhnung beispielsweise den grössten Einfluss auf das Mortalitätsrisiko (36\% Risikoreduktion) und liegt damit über der Wirkung, die je mit der Einnahme von Statinen (29\%), von Aspirin (20\%), von Betablockern (23\%) oder von ACE-Hemmern (23\%) erreicht wird [5].

Nimmt man die Unfälle hinzu, so führten diese Krankheiten und Risikofaktoren im Jahr 2003 zu direkten Kosten von rund 17 Milliarden Franken (Tabelle 1), was mehr als einem Viertel der Gesundheitskosten der Schweiz entspricht [6]. Und selbst wenn die Zahlen der Tabelle 1 manchmal Extrapolationen von Daten aus kleinen Stichproben darstellen, vor allem bei den stressbedingten Krankheiten, so lässt sich doch festhalten, dass eine bessere Prävention die Sterblichkeit gesamtschweizerisch erheblich senken würde [6-9]. Laut einem 2006 von der OECD und WHO publizierten Bericht wendet die Schweiz im Vergleich mit den anderen OECD-Ländern jedoch wenig Mittel für Prävention und Gesundheitsförderung auf [10].

Während die Wirksamkeit einiger präventiver Interventionen (Tabakentwöhnung) und von Screeningtests (Nachweis von okkultem Blut im Stuhl, Mammographie) erwiesen ist, so fehlt dieser Nachweis (noch?) bei anderen Massnahmen (PSA, Spiral-CT, Screening von arteriosklerotischen Placques) [11]. Beschliesst ein Arzt, einen Screeningtest durchzuführen, so wendet er sich an eine - per definitionem - asymptomatische Person; sein Verhalten wird also durch wichtige

\section{Abbildung 1}

Hauptsächliche Todes- und Krankheitsursachen in der Schweiz [1-4].
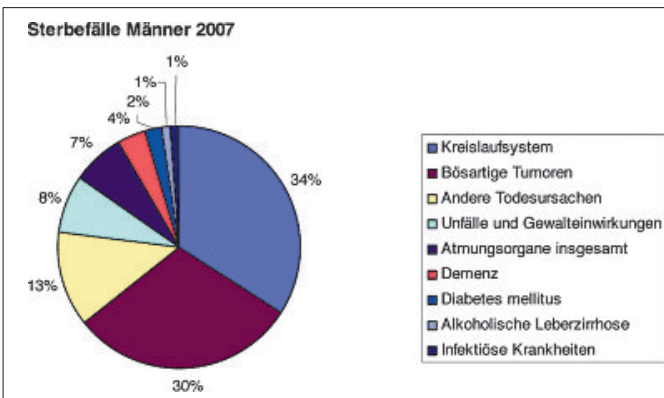

Verlorene potentielle Lebensjahre Mănner 2007

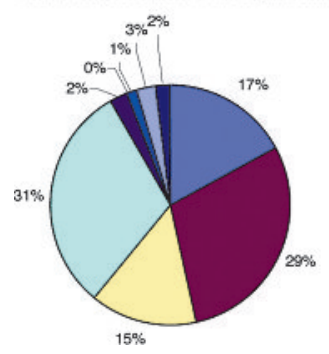

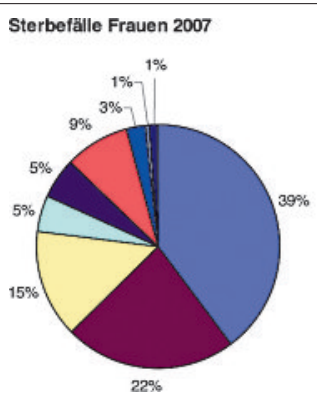

\section{Kreislautsystem} - Bösartige Tumoren a Andere Todesursachen 口 Untalle und Gewalteinwirkungen - Atmungsorgane insgesamt - Demenz - Diabetes mellitus alkoholische Leberzimnose - Intektiöse Krankheiten

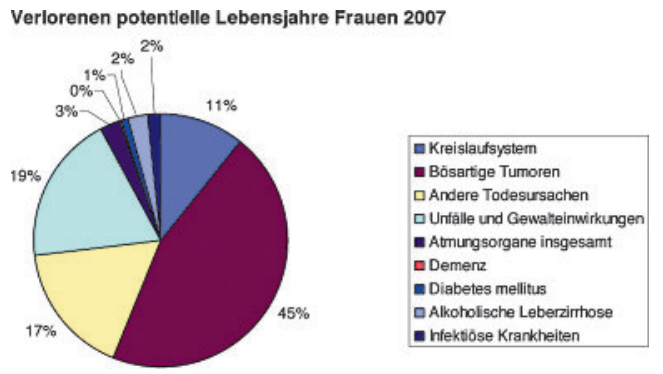




\section{Tabelle 1}

Direkte und indirekte Kosten von ausgewählten beeinflussbaren Krankheiten und Unfällen bzw. Risikofaktoren in Milliarden Franken pro Jahr (2003) [6].

\begin{tabular}{|c|c|c|c|c|}
\hline & $\begin{array}{l}\text { direkte } \\
\text { Kosten }\end{array}$ & $\begin{array}{l}\text { indirekte } \\
\text { Kosten }\end{array}$ & $\begin{array}{l}\text { Total direkte } \\
\text { und indirekte } \\
\text { Kosten }\end{array}$ & $\begin{array}{l}\text { direkte } \\
\text { Kosten in \% } \\
\text { Gesundheits } \\
\text { kosten }\end{array}$ \\
\hline $\begin{array}{l}\text { Berufs- und Nichtberufsunfälle } \\
\text { (ohne Verkehr) }\end{array}$ & 4,1 & 14,2 & 18,3 & 8,2 \\
\hline Psychische Krankheiten & 2,9 & 5,5 & 8,4 & 5,8 \\
\hline Tabakbedingte Erkrankungen & 1,6 & 4,1 & 5,7 & 3,2 \\
\hline $\begin{array}{l}\text { Stress bei der berufstätigen } \\
\text { Bevölkerung }\end{array}$ & 2,3 & 3,1 & 5,4 & 4,6 \\
\hline Verkehrsunfälle & 3,8 & 0,5 & 4,2 & 1,0 \\
\hline $\begin{array}{l}\text { Übergewichtbedingte } \\
\text { Erkrankungen }\end{array}$ & $1,2-1,7$ & $1,1-1,7$ & $2,3-3,4$ & 2,9 \\
\hline Alkoholbedingte Erkrankungen & 0,85 & 1,6 & 2,5 & 1,7 \\
\hline Total & $16,8-17,3$ & $30,1-30,7$ & $46,8-47,9$ & 27,4 \\
\hline
\end{tabular}

ethische Überlegungen beeinflusst, denn «früher» bedeutet nicht immer «besser». Ein Artikel im «Lancet» wies kürzlich darauf hin: «Screening can promote or impair health, depending on its application» [12]. Es erscheint heute daher opportun, Ärztinnen und Ärzten Instrumente zur Verfügung zu stellen, mit denen sie zusammen mit dem Patienten die Ungewissheiten im Zusammenhang mit einem Screening diskutieren können. Die Arztpraxis stellt dabei einen besonders geeigneten Ort für die Umsetzung einer zugleich effizienten und kostenbewussten Präventions- und Gesundheitsförderungspolitik dar [13-17].

\section{Ziele von EviPrev}

EviPrev liefert einen umfassenden Ansatz für Prävention und Gesundheitsförderung in der Hausarztpraxis. Die empfohlenen Massnahmen stützen sich auf wissenschaftliche Daten.

EviPrev besteht aus sechs Elementen: 1) Information der Ärztinnen und Ärzte über nachgewiesenermassen effiziente Präventionsmassnahmen; 2) Fortbildung für Hausärztinnen und Hausärzte, um deren Kenntnisstand und Kompetenzen im Bereich der Präventivmedizin zu erhalten und verbessern; 3) Hilfsmittel zur Umsetzung des Programms in der Hausarztpraxis (Tools); 4) Hilfsmittel zur Unterstützung der Patientinnen und Patienten bei der Änderung ihres Gesundheitsverhaltens; 5) Information der Öffentlichkeit zur Stärkung der Gesundheitskompetenz (health literacy); 6) eine wissenschaftliche Evaluation.

Die Umsetzung sieht zwei strategische Achsen vor: zum einen EviPrev-P als Papierversion und zum andern EviPrev-IT als elektronische Version zur Entscheidungshilfe (decision support). Ein neues, innovatives Element von EviPrev-P ist die «Präventionskarte». Sie lehnt sich an den Impfausweis an und könnte je nach Ergebnissen einer Pilotphase erst auf kantonaler, dann auf nationaler Ebene eingeführt werden. Sie soll Arzt und Patient als lebenslanges Hilfsmittel dienen. Sie wird durch weitere Instrumente ergänzt: Hilfsmittel für das ärztliche Gespräch und die gemeinsame Entscheidungsfindung, Informationsmaterial für die Patientinnen und Patienten wie etwa Broschüren und Infoblätter.

Die Papierversion des Programms lässt sich breit einsetzen, kann aber das volle Potential von EviPrev in der Hausarztpraxis nicht ausschöpfen. Aus diesem Grund wird parallel dazu die Version EviPrev-IT entwickelt. EviPrev-IT basiert auf den neuen Informations- und Kommunikationstechnologien und orientiert sich an der Zukunftsvision der eHealth-Strategie des Bundes [18]. Spezielle Funktionen sollen die Patientinnen und Patienten unterstützen, wo nötig ihr Gesundheitsverhalten zu ändern. Als echtes computergestütztes Decision-Support-System unterstützt EviPrev-IT Ärztinnen und Ärzte in ihrer Rolle als Beratende, insbesondere bei der Steuerung einer individuell angepassten, kohärenten und evidenzbasierten Prävention.

\section{Die wichtigsten Leistungen von EviPrev:}

- Bereitstellung von Empfehlungen für evidenzbasierte individuelle Präventionsmassnahmen.

- Erstellen des individuellen Risikoprofils des Patienten und Evaluation des Risikosenkungspotentials unter Zuhilfenahme der Instrumente von EviPrevIT.

- Lieferung von Unterlagen zur Verbesserung der Gesundheitskompetenz der Bevölkerung (Health Literacy), insbesondere auf Grundlage des Konzepts des Shared Decision Making.

- Unterstützung von Arzt und Patient bei der Umsetzung der gemeinsam vereinbarten Massnahmen und der Verhaltensänderungen des Patienten (Interaktivität, Möglichkeit von Feedbacks) mit Vorschlägen zu Kontrollintervallen für die individuelle Begleitung unter Berücksichtigung der Ziele und der Ausgangssituation.

\section{Vorgehen}

Die Lancierung von EviPrev ist für 2012 geplant, nach einer Pilotphase in den Jahren 2010-2011. Das Projekt integriert die Meinungen der schweizerischen Hausärztinnen und Hausärzte, insbesondere auf Grundlage der Studie «Focusprev», einer von der PMU, dem IUMSP Lausanne und den Instituten für Allgemeinmedizin der Universitäten Zürich und Lausanne durchgeführten qualitativen Untersuchung, die von der Schweizerischen Akademie der Medizinischen Wissenschaften unterstützt wurde. Diese Studie hatte zum Ziel, mittels Fokusgruppen von in der Deutschschweiz und in der Romandie niedergelassenen Ärztinnen und Ärzten deren Erwartungen sowie mögliche Hindernisse im Bereich der Präventivmedizin zu eruieren [19]. Die ersten Ergebnisse zeigen, dass die Ärztinnen und Ärzte präventive Interventionen als Teil ihrer Aufgabe auffas- 
sen und Empfehlungen erwarten, die zwar evidenzbasiert sind, aber gleichzeitig unabhängig von der Pharmabranche und die eine individuelle Beratung unter Einbezug der spezifischen Bedingungen eines Patienten (klinische Komplexität, Co-Morbiditäten) und der ambulanten Praxis (finanzielle und praktische Einschränkungen) erlauben.

Während und nach der Lancierung wird aktiv für die Prävention und Gesundheitsförderung in der Hausarztpraxis geworben. Die Informationsmittel zur Verbesserung der Gesundheitskompetenz von Patientinnen und Patienten werden in allen Praxen gestreut und die Ärztinnen und Ärzte eingeladen, sie systematisch ihren Patientinnen und Patienten abzugeben. Ärztinnen und Ärzte werden auch zu den Fortbildungsveranstaltungen eingeladen. Gestützt auf die Evaluation ist in einer zweiten Phase auch geplant, das Konzept von EviPrev in den Ausbildungsgang für Medizinstudierende aufzunehmen, zumindest an einigen medizinischen Fakultäten.

EviPrev-IT wird gleichzeitig mit EviPrev-P eingeführt. Spezifische Kommunikations- und Verbreitungsmassnahmen sollen eine möglichst breite Anwendung sicherstellen. Ärztenetzwerke sind eine bevorzugte Zielgruppe. Die Entwicklungen betreffend die e-HealthStrategie des Bundes sollen mit der Absicht verfolgt werden, EviPrev dort als festes Modul für die Prävention zu integrieren. Abbildung 2 gibt einen Überblick über Etappen und Elemente von EviPrev.

\section{Rechtlicher und regulatorischer Bezugsrahmen}

Die Schweiz verfügt noch über kein Präventionsgesetz. Gemäss den Empfehlungen der Fachkommission «Prävention + Gesundheitsförderung» hat das Departement des Innern im Jahr 2007 einen Entwurf eines Präventionsgesetzes erarbeitet [20]. Nach einer breiten Vernehmlassung 2008-2009 hat der Bundesrat die Botschaft und den Entwurf für ein Präventionsgesetz vor kurzem ans Parlament überwiesen, und die Beratun-

Abbildung 2

Outcome EviPrev.

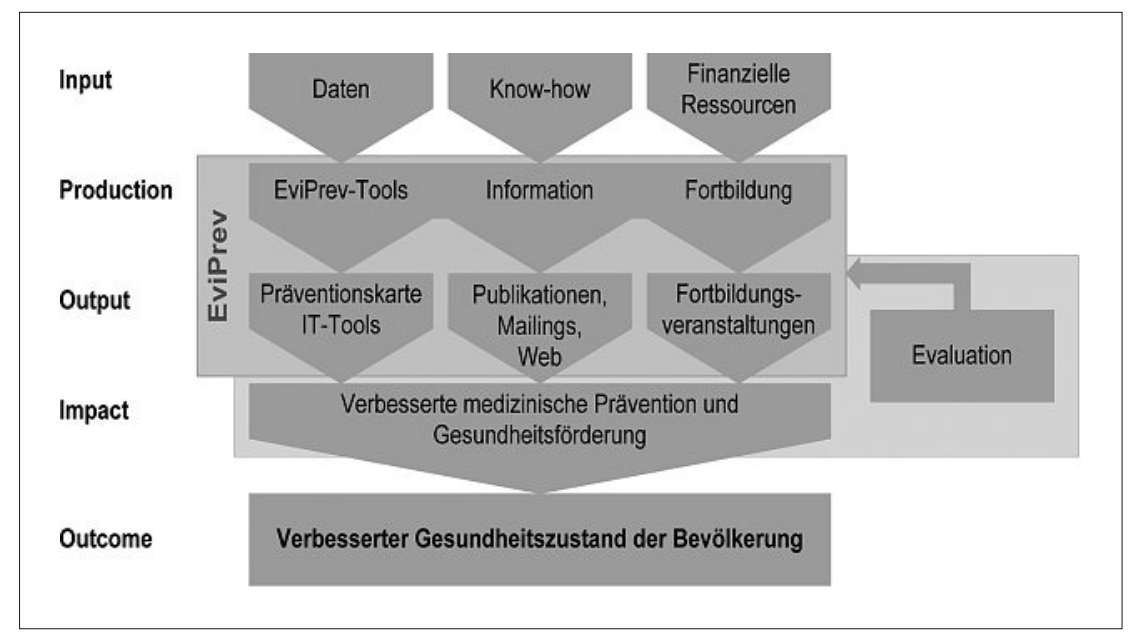

gen in den Gesundheitskommissionen des Nationalrates werden 2010 anlaufen. Eine solche Rahmengesetzgebung könnte auch die medizinische Prävention umfassen und damit eine zentrale Grundlage für EviPrev bilden. Durch geltende Bestimmungen des Krankenversicherungsgesetzes (KVG) und darauf gestützte Regelungen ist ein Teil der EviPrev-Interventionen bereits kassenpflichtig. Im Zuge der erwähnten Neuregelung soll darauf hingewirkt werden, dass bestehende Deckungslücken möglichst geschlossen werden können.

\section{Trägerschaft und Organisation}

Die Lancierung von EviPrev stützt sich auf eine breite Trägerschaft mit Vertretern der verschiedenen beteiligten Sektoren: Hausärzte, Fachärzte und Public HealthFachleute. Entwickelt wird das Programm EviPrev von der Policlinique Médicale Universitaire Lausanne (wissenschaftliche Grundlagen und Fortbildung), in Zusammenarbeit mit dem Institut für Hausarztmedizin der Universität Zürich (praxisgerechte Umsetzung und Evaluation), der Arbeitsgruppe Prävention des Kollegiums für Hausarztmedizin (KHM) (praxisgerechte Umsetzung), dem CC HNE Kompetenzzentrum Health Network Engineering der Universität St. Gallen (Strategie eHealth und IT) und PHS Public Health Services (Projektmanagement und Kommunikation). In dieser Perspektive haben die Partner 2008 den Verein EviPrev geschaffen und das Bundesamt für Gesundheit (BAG) hat kürzlich einen finanziellen Beitrag geleistet. Um das Projekt breit $\mathrm{zu}$ verankern und Synergien $\mathrm{zu}$ nutzen, bestehen Partnerschaften mit verschiedenen Stakeholdern aus dem Gesundheitswesen. Bisher wird das Projekt EviPrev ideell unterstützt durch das BAG, die Schweizerische Konferenz der kantonalen Gesundheitsdirektorinnen und Gesundheitsdirektoren (GDK), Gesundheitsförderung Schweiz, die bfu - Beratungsstelle für Unfallverhütung, die Krebsliga Schweiz, die Rheumaliga Schweiz und die Schweizerische Diabetes-Gesellschaft. Mit Organisationen der Ärzteschaft und weiteren NGOs (Non Governmental Organizations) werden gegenwärtig Gespräche geführt, um die Trägerschaft von EviPrev gezielt zu erweitern. Kann das geplante Vorgehen eingehalten werden, so erfolgt die Finanzierung mittels Geldern der öffentlichen Hand, von Stiftungen, NGOs und mit privatem Kapital.

EviPrev will den schweizerischen Ärztinnen und Ärzten in Übereinstimmung mit internationalen Empfehlungen einen umfassenden Ansatz im Bereich der Prävention und neue Instrumente für ihre präventiven Interventionen in der Praxis liefern. Das Programm ergänzt das Projekt «Gesundheitscoaching» des Kollegiums für Hausarztmedizin [21]. Die praktische Umsetzung (Anpassung an die Realitäten in der Arztpraxis) sowie die Evaluation der Wirksamkeit werden durch eine Gruppe wahrgenommen, die sich aus Vertreterinnen und Vertretern der PMU Lausanne und der fünf Institute für Hausarztmedizin zusammensetzt. 


\section{Literatur}

1 BFS. Bundesamt für Statistik. Sterbefälle und Sterbeziffern wichtiger Todesursachen, nach Alter, Männer (je-d-14.02.05.01). 2009 [cited 13.11.2009]; Available from: www.bfs.admin.ch/bfs/portal/de/ index/themen/14/02/04/key/01.html

2 BFS. Bundesamt für Statistik. Sterbefälle und Sterbeziffern wichtiger Todesursachen, nach Alter, Frauen (je-d-14.02.05.01). 2009 [cited 13.11.2009]; Available from: www.bfs.admin.ch/bfs/portal/de/ index/themen/14/02/04/key/01.html

3 BFS. Bundesamt für Statistik. Verlorene potentielle Lebensjahre, Männer (je-d-14.02.05.06). 2009 [cited 13.11.2009]; Available from: www.bfs.admin.ch/bfs/ portal/de/index/themen/14/02/04/key/01.html

4 BFS. Bundesamt für Statistik. Verlorene potentielle Lebensjahre, Frauen (je-d-14.02.05.07). 2009 [cited 13.11.2009]; Available from: www.bfs.admin.ch/bfs/ portal/de/index/themen/14/02/04/key/01.html

5 Crichley JA, CS. Mortality risk reduction associated with smoking cessation in patients with coronary heart disease: a systematic review. JAMA. 2003(290): 86-97.

6 Berset C, Lehmann P. Etude comparative des coûts sociaux des principaux déterminants de morbidité et mortalité évitables en Suisse (internes Dokument) Bern: Bundesamt für Gesundheit; 2004

7 Obrist R, Schopper D. Nationales Krebsprogramm für die Schweiz 2005-2010. Bern: Oncosuisse; 2005.

8 Smala A, Beeler I, Szucs TD. Die Kosten der körperlichen Inaktivität in der Schweiz. Universität Zürich: Institut für Sozial- und Präventivmedizin; 2001.

9 Commission of the European Communities, Promoting healthy diets and physical activity: a European dimension for the prevention of overweight, obesity and chronic diseases (Green Paper). Brussels: 2005.

10 OECD. Organisation for Economic Co-operation and development and WHO, World Health Organisation. OECD-Berichte über Gesundheitssysteme: Schweiz. Paris: OECD; 2006.
11 US Preventive Services Task Force. Active Recommendations (on preventive measures, including screening tests, counseling, immunizations, and preventive medications). 2006 (cited 15.10.2006); Available from: www.ahrq.gov/clinic/uspstfix.htm

12 Grimes DA. Uses and abuses of screening tests. Lancet. 2002;(359): 881-4.

13 Neuner S. Prävention und Gesundheitsförderung in der Hausarztmedizin: Eine unserer Kernkompetenzen! Primary Care. 2006;6(23):420_1.

14 Cornuz J, DiCarlantonio D, Pécoud A, Paccaud F. Physician attitudes toward prevention: Importance of intervention-specific barriers and phycisian health status. Family Practice. 2000;(17):536-40

15 Rodondi $\mathrm{N}$ et al. Therapy Modifications in Response to Poorly Controlled Hypertension, Dyslipidemia and Diabetes Mellitus. Annals of Internal Medicine. 144(7):475-84.

16 Cornuz J, Guessous I, Rodondi N. Prévention primaire et dépistage chez l'adulte: mise à jour 2006. Revue Médicale Suisse. 2006; 2(50):262-73.

17 Maciosek MV et al. Priorities Among Effective Clinical Preventive Services - Results of a Systematic Review and Analysis. American Journal of Preventive Medicine. 2006; 31(1):52-61.

18 BAG. Bundesamt für Gesundheit. Strategie «eHealth» Schweiz. Bern: 2007.

19 Zyska Cherix A, Moix E, Meili M, Neuner S, Cornuz J, Spencer B. Perceptions and barriers to the implementation of prevention and health promotion in General Practice: a Study of Swiss GPs. In WONCA 2009. Basel: 2009.

20 EDI. Fachkommission «Prävention + Gesundheitsförderung», Bericht «Zukunft von Prävention und Gesundheitsförderung in der Schweiz». Bern: Eidg. Departement des Innern; 2006.

21 Grüninger U, Hösli R, Neuner S, Schmid M. Gesundheitscoaching - ein Programm für Gesundheitsförderung und Prävention in der Hausarztpraxis vom Kollegium für Hausarztmedizin (KHM). Schweiz Ärztezeitung. 2009;90(45):1729-32.

\section{Sie lesen gerade eine Zeitschrift des Schweizerischen Ärzteverlags EMH}

\section{Wussten Sie schon,}

- dass EMH ein Gemeinschaftsunternehmen der Verbindung der Schweizer Ärztinnen und Ärzte FMH und der Schwabe AG ist, dem mit Gründung 1488 ältesten Druck- und Verlagshaus der Welt?

- dass EMH mit insgesamt zehn Fachzeitschriften, einem umfangreichen Online-Angebot sowie einem wachsenden Buchprogramm der führende Verlag für medizinische Zeitschriften in der Schweiz ist?

dass sämtliche bei EMH erscheinenden Zeitschriften offizielle Publikationsorgane der jeweils zuständigen medizinischen Fachorganisationen sind?

Wenn Sie mehr über EMH wissen möchten, finden Sie unter www.emh.ch weitere Informationen. 